\title{
Clinical research in the emergency department conducted by non-emergency physicians: potential problems and proposed recommendations
}

\author{
Andrew Worster, MD, MSc, FCFP; Brian H. Rowe, MD, MSc, FCCP; Ian G. Stiell, MD, MSc, FRCPC; \\ Bjug Borgundvaag, PhD, MD; Marco L.A. Sivilotti, MD, MSc, FRCPC, FACEP, FACMT; \\ Sam G. Campbell, MB, BCh, Dip PEC(SA); Christopher M.B. Fernandes, MD; Duncan S. Mackey, MD; \\ Karen Woolfrey, MD, FRCPC, FACEP; Riyad B. Abu-Laban, MD, MHSc, FRCPC; \\ Jacques S. Lee, MD, MSc, FRCPC
}

\section{Introduction}

The emergency department (ED) is often seen as a desirable place to conduct clinical research due to the broad and undifferentiated spectrum of acute conditions encountered. As a result, ED administrators are often faced with requests for patient access by non-ED researchers. Conflict may arise between these 2 groups regarding which studies should be conducted in the ED. Non-emergency (outside) researchers may perceive that their institutional affiliation, and the overhead fees charged by their hospital afford them the "right" to conduct clinical research in the ED, while ED administrators may counter that it is their responsibility to evaluate research protocols with respect to their effects on patient care, work-flow, and resources.

The challenges of conducting clinical research in the ED may not be fully appreciated by those not regularly working in this environment. The constant re-evaluation of priorities, and the necessity for clinical care to take precedence over research, makes conducting clinical studies difficult. Unless consideration is given to staff education and facilitating patient enrolment when designing clinical studies, even motivated ED staff may not enrol patients.

The purpose of this paper is to explore the key issues of concern when non-emergency-based researchers wish to conduct clinical studies in the ED.

\section{Ethical and financial considerations}

Research ethics board (REB) approval - Ideally, no ED-based study would be considered by the REB without prior authorization from ED administrators. In many institutions however, consultation with ED administration is not required prior to REB submission of ED-based studies. Moreover, many sites do not have emergency medicine representation on the REB, which limits the ability to understand the problems associated with conducting studies in the ED setting. ' Once a trial has been deemed to be "safe" for the institution's patients, there might be a sense that the ED should cooperate with outside investigators.

We do not believe that an ED should feel obligated to participate in a study because it is approved by the REB.

From Hamilton Health Sciences and McMaster University, Hamilton, Ont. (Worster and Fernandes); the University of Alberta, Edmonton, Alta. (Rowe and Mackey); Capital Health, Edmonton (Rowe); Ottawa Health Research Institute and the University of Ottawa, Ottawa, Ont. (Stiell); Mount Sinai Hospital and the University of Toronto, Toronto, Ont. (Borgundvaag); Queen's University, Kingston, Ont. (Sivilotti); Dalhousie University and Queen Elizabeth II Health Sciences Centre, Halifax, NS (Campbell); Lethbridge Regional Hospital, Lethbridge, Alta. (Mackey); Sunnybrook and Women's College Health Sciences Centre and the University of Toronto, Toronto, Ont. (Lee); St. Joseph's Hospital and McMaster University, Hamilton, Ont. (Woolfrey); and the University of British Columbia and the Centre for Clinical Epidemiology \& Evaluation, Vancouver, BC (Abu-Laban).

Received: Sept. 22, 2004; final submission: May 14, 2005; accepted: May 19, 2005

This article has been peer reviewed.

Can J Emerg Med 2005;7(4):241-8 
The care of ED patients must not be compromised by clinical studies regardless of authorization from non-ED groups. This is in keeping with the "Research" section of the Canadian Medical Association's Code of Ethics, ${ }^{2}$ which states:

38. Ensure that any research in which you participate is evaluated both scientifically and ethically and is approved by a research ethics board that meets current standards of practice.

39. Inform the potential research subject, or proxy, about the purpose of the study, its source of funding, the nature and relative probability of harms and benefits, and the nature of your participation including any compensation.

40. Before proceeding with the study, obtain the informed consent of the subject, or proxy, and advise prospective subjects that they have the right to decline or withdraw from the study at any time, without prejudice to their ongoing care.

Recommendation: All studies aiming to recruit patients in the ED should be approved by a duly constituted REB, receive ED administrative review and approval, and be deemed to not interfere with the provision of high quality care.

Potential solution: ED administration should engage the REB and hospital administration in discussions to achieve ED representation in the approval of all research protocols that involve ED patients. Achieving an institutional consensus among ED administrators on this principle is a vital first step in achieving this goal.

Disclosures - Financial considerations are a common reason for researchers to participate in industry-sponsored trials, and investigators may not fully disclose the level of trial funding to ED administrators. Compared to investigators for trials sponsored by peer-reviewed granting agencies, investigators in industry-sponsored studies frequently receive considerably larger payments. According to the Tri-Council Policy Statement on the Ethical Conduct for Research Involving Humans, all potential conflicts of interest must be disclosed to the REB and the patient. ${ }^{3} \mathrm{We}$ feel this information should also be disclosed to ED administrators who are evaluating the suitability of a trial for conduct in the ED.

Many academic EDs have policies in place that guarantee the ED a portion of the funding for industry-sponsored trials. One prominent academic institution requires a minimum payment of \$200/patient enrolled. From an informal email survey of academic Emergency Medicine Research Directors conducted in the Spring of 2004, it was concluded that a minimum rate of $10 \%-25 \%$ of the enrolment fee should be shared with the ED for conducting industrysponsored outside research studies, for these trials would not be possible without the cooperation and participation of ED staff. This fee may be waived at the discretion of the ED administration in cases where study funding arises from non-profit organizations (e.g., governments, foundations, charities).

Remuneration - The CMA's Policy Physicians and the Pharmaceutical Industry (Update 2001) ${ }^{4}$ states in the "Industry-sponsored research" section:

11. It is acceptable for physicians to receive remuneration for enrolling patients or participating in approved research studies only if such activity exceeds their normal practice pattern. This remuneration should not constitute enticement.

This is consistent with the World Medical Association's Declaration of Helsinki, which precludes physicians from receiving any material benefit for referring patients to industry-sponsored studies. ${ }^{5}$ In areas of uncertainty, the local REB should be able to provide guidance, as most REB applications require a description of recruitment methods for any trial.

Recommendations: There should be mandatory disclosure of full study budget details and conflicts of interest including a site-specific allocation of funding, incentives to the investigators, disclosure of speaking fees, consultant fees, and stock ownership to the local REB. The study budget including payments to investigators should be made available to ED administrators when considering participation in non-emergency-based studies. There should be sharing of financial benefits at an appropriate and mutually agreeable rate. Residual funds should be used for academic activities such as group education or emergency-based research.

Potential solutions: The formal process recommended above for all research studies should include provisions for disclosure of conflicts of interest budget details, and financial collaboration.

Consent - Obtaining informed consent is a critical step in the execution of any clinical research study. In order for consent to be truly informed, the prospective study participant must be provided with all the information needed to make a decision regarding participation in the study. Studies originating from outside researchers may involve interventions or treatments/therapeutics which are beyond the scope of ED practice, and which ED personnel who are not investigators may not fully understand. This may be an even greater issue when ED nurses or residents obtain consent.

Recommendation: The individual obtaining consent must 
be able to explain the study to prospective participants in a language and at a level that they understand, in a manner that avoids coercion or undue influence. Furthermore, this individual must be sufficiently knowledgeable of both the study and the field of research to clearly explain the implications of participation, including possible risks.

Potential solution: Potential ED co-investigators should be identified at the planning stage of any clinical study that intends to enrol patients in the ED setting. Involvement of ED personnel in the early stages of a clinical study will increase the likelihood that ED staff will be familiar with both the area of research, and the implications of study participation.

\section{Liability}

EDs and their staff may assume liability by participating in studies. The potential risk is usually greatest with therapeutic studies, while observational studies often have minimal or no risk. Many industry-sponsored studies provide liability insurance. It is the responsibility of the hospital/institution lawyers to ensure the adequacy of the indemnity. The primary responsibility of hospital lawyers is to protect the hospital so that coverage may not extend to investigator and non-investigator physicians participating in the care of study subjects. Furthermore, physicians should not assume they have coverage from their own malpractice insurance for participation in research. The Canadian Medical Protective Association (CMPA) states that physicians are generally covered for Phase III and IV studies; however, some debate exists about the liability coverage associated with Phase I/II studies. ${ }^{6}$

Recommendation: Studies should not place ED staff at increased risk of liability; liability coverage and indemnity clauses should be examined closely.

Potential solution: Canadian emergency researchers should be unanimous in requiring adequate liability insurance from industry sponsors for research activities that fall outside of CMPA coverage.

\section{Practical considerations and feasibility issues}

Provision of care - ED administrators should support outside research requests provided care will not be compromised for either the study population or other ED patients. Since priority must be given to the most acutely illpatients, investigators should commit to providing timely, comprehensive care to all patients regardless of their eligibility for research participation. Furthermore, when a pa- tient returns with a complication related to the study, the investigators should assume responsibility and care for the patient in keeping with CMA policy: Consider first the well-being of the patient. ${ }^{2,4}$

Recommendation: Investigators should only be approved to conduct studies on patients for whom they are prepared and available to assume the care of in the event of study related complications.

Potential solution: ED administration should engage the REB and hospital administration in discussions to achieve ED representation in the approval of all research protocols that involve ED patients.

Impact on emergency department operations - Study protocols that either facilitate or, at most, minimally impact patient care are more likely to be successfully completed in the ED. Studies that involve ED resource utilization in excess of what patients would typically receive in the course of their care would impact heavily on most EDs, and are more likely to encounter obstacles or fail. Nonemergency researchers often underestimate, or fail to appreciate, the impact of studies on ED care and throughput. Inappropriate ED resource utilization is also common with underfunded, internally funded, or student/resident projects. It may be helpful when designing studies to involve emergency physicians as co-investigators thereby increasing the chances of successfully completing the research. The impact of study operations (e.g., obtaining consent, drawing blood work and other tests, managing complications) is best assessed by ED personnel.

Recommendation: Clinical studies should not increase the net workload or resource utilization of the ED. Departments should have the authority to decline studies for operational and workload reasons. In all cases, the study budget should reimburse the ED for any additional costs irrespective of fees paid to the institution.

Potential solutions: Involvement of ED personnel in the planning of clinical studies intended to be conducted in the ED setting may increase the chances of the study being successfully completed, and minimize the impact of the study on the day-to-day operations of the ED.

Study duration - Anticipating the rate of patient accrual can be very difficult, since this depends on how many eligible patients present, are referred, and consent to participate. Investigators often underestimate the time needed to enrol the required sample size. This commits ED staff to participating for longer periods than anticipated. Con- 
versely, industry sponsors may terminate a trial early for a variety of reasons, leaving the ED with financial obligations to employees.

Recommendations: If possible, a feasibility (pilot) study should be conducted prior to the commencement of any clinical study. The enrolment estimates of a proposed study protocol should be assessed by ED administrators prior to study approval. Agreed study duration limits should be placed on all clinical studies along with stipulations and conditions for premature termination of the study. Extensions should not occur without a formal approval by ED administration.

Potential solutions: In all industry studies, a formal contract should be executed between the corporate sponsor, the institution and the investigator. Where possible, funding should be obtained from industry sponsors to collect pilot data prior to committing to participation in a study.

Competing / Multiple studies - Some patient populations are in greater demand for studies because of trends or local investigator interest or expertise. Studies competing for the same patient population will reduce the number of patients enrolled and should generally be discouraged. Poor enrolment delays the completion of a trial, and may lead to a clinical study being halted at a given institution. Situations where patients are repeatedly approached to participate in multiple studies during their ED visit should also be avoided.

Recommendation: Additional studies should not be approved for the ED if potentially competing for the same patient population.

Potential solutions: A policy should be established where subjects are asked no more than once per ED visit to participate in any investigational study.

Collaboration considerations - We define "collaboration" on outside research as involving intellectual (planning and approval), academic (publications authorship and credit), operational (resources) and financial (costs overages and deficits) issues. Collaboration is demonstrated by a group of clinicians/scientists sharing in the development, implementation, evaluation, and academic aspects, including publication, of a research project.

Academic achievement will advance both individual careers and the discipline of emergency medicine. Many emergency personnel are interested in participating in clinical research studies for these reasons alone. Being remunerated for time spent enrolling patients does little to ac- complish either of these goals, regardless of the amount paid. Academic collaboration is, therefore, an important inducement to ED staff for contributing to research studies.

Critical to the research process is an in-depth knowledge of both the clinical condition under study, and the research question. Likewise, planning a clinical study requires an in depth understanding of the care for the involved patients at all stages of their ED visit \pm hospitalization. We feel strongly that when non-ED researchers include ED staff as co-investigators several positive benefits occur. Both parties gain a better understanding of the challenges faced by their colleagues at their respective stages of care for involved patients. Second, ED staff may gain new or more current knowledge regarding advances in caring for patients with the condition under study.

ED staff can more effectively publicize and promote studies while investigators may be more limited. For example, emergency and critical care experts at one ED conducted a RCT on the use of hirudin in acute coronary syndromes. EPs were involved closely in patient recruitment and treatment, named as co-investigators, and their research group received funding allowing them to further other research endeavors. The model was so successful that the investigator group was ranked first in North America for recruitment as well as quality of reporting. This model leads to more efficient research and better institutional benefits (e.g., site reputation, financial and future research opportunities). Such arrangements may well assist EDs in enhancing academic productivity and improving links between different research groups within an institution.

Recommendation: Collaborative models are the ideal means for conducting outside research in the ED. As a minimum, an EP should be listed as a co-investigator and involved with the ED planning and design phase of the study. Authorship credit could follow, provided that the EP satisfies the standard authorship requirements.

Potential solutions: The formal process for all research studies should include provisions for collaboration with ED researchers/staff.

Formal application process - Despite the best intentions, disagreements may occur between the ED and investigators prior to or during a study. One strategy to avoid conflict or misunderstandings is to require completion of a specific ED Research Application form tied to a review process that incorporates the previously listed recommendations and a designated "Research Committee" or "Research Advisory Committee." Such committees should include ED staff with administrative, clinical, and research 
responsibilities. Additional support and formalization of this process can be obtained through the institution's Medical Advisory Committee (MAC).

Recommendations: EDs are encouraged to create site-specific application forms and processes for research access to the ED incorporating the previously outlined components.

Potential solutions: Two sample application forms and an MAC policy statement are provided in Appendix 1, Appendix 2 and Appendix 3, respectively. Sites are encouraged to revise these to suit their local approaches.

\section{Conclusion}

The ED is a desirable setting for clinical research. Both academic and non-academic EDs are faced with numerous requests for non-ED-directed research, creating the potential for significant problems to arise. We feel that most, if not all, of these difficulties can be minimized or eliminated if a collaborative approach to conducting these studies is adopted. This paper is not intended to provide an exhaustive list of problems that can arise when outside researchers attempt to conduct clinical research studies in the $\mathrm{ED}$, but rather to provide the combined experience of the authors surrounding some of the most common issues encountered. The suggested solutions have worked in our settings and have allowed outside research studies to be completed within our EDs.

EDs and their staff, while remaining supportive of research endeavours for the advancement of knowledge and care, must be vigilant in reviewing and approving external studies in order to protect patients, staff and the institution. An organized, standardized approach by EDs to deal with the possible problems outlined above will lead to increased clarity, collaboration and research success for all parties.
Acknowledgements: Dr. Rowe is supported by the Government of Canada (Canadian Institutes of Health Research [CIHR]) as a Research Chair. Dr. Stiell is a Distinguished Investigator of the CIHR. Dr. Borgundvaag is supported by the Fogel Foundation. Dr. Abu-Laban is supported by a Clinical Scholar Award from the Michael Smith Foundation for Health Research.

Competing interests: None declared.

Key words: emergency department; clinical trials; clinical research; collaboration

\section{References}

1. Reed CR, Camargo CA Jr. Recent trends and controversies in industry-sponsored clinical trials. Acad Emerg Med 1999; 6(8):833-9.

2. CMA Code of Ethics [policy]. CMAJ 2005;172(8):1053-5. Available: www.cma.ca/index.cfm/ci_id/2419/la_id/1.htm (accessed 2005 May 30).

3. Tri-Council Policy Statement: Ethical conduct for research involving humans. Section 4: Conflict of interest; Article 4.1.A. Available: www.ncehr-cnerh.org/english/code_2/sec04.html (accessed 2005 May 30).

4. CMA Policy. Physicians and the pharmaceutical industry (update 2001). Ottawa: Canadian Medical Association. 2001. Available: www.cma.ca//multimedia/staticContent/HTML /N0/12/where_we_stand/physicians_and_the_pharmaceutical_in dustry.pdf (accessed 2005 May 30).

5. World Medical Association Declaration of Helsinki. Available: www.wma.net/e/policy/b3.htm (accessed 2005 June 3).

6. Clinical research: Will the CMPA help me? CMPA Information Lett 2003;18(2).

7. Canadian Association of Emergency Physicians Web site [Internet]. Ottawa: The Association. Canadian Journal of Emergency Medicine Policies and Procedures. 5. Criteria for Authorship. Available: www.caep.ca/004.cjem-jcmu/004-00.cjem/004-01iibe.cjem-policies.htm\#criteria (accessed 2005 May 30).

Correspondence to: Dr. Andrew Worster, Emergency Department, McMaster University Medical Centre, 1200 Main St. W, Hamilton ON L8N 3 Z5

See Appendices, pages 246, 247 and 248. 


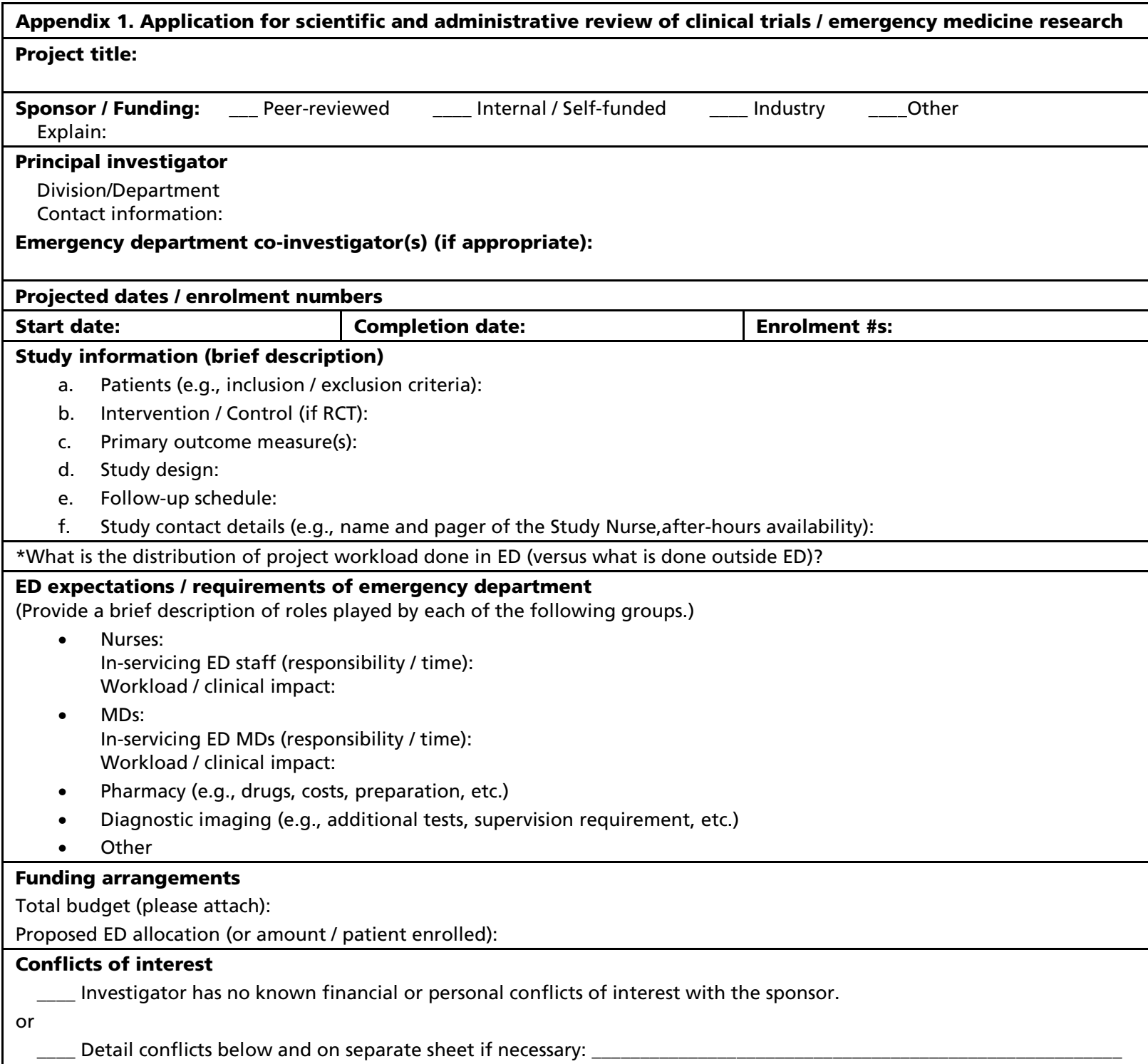

\section{Study approval}

We accept the proposal as outlined by the investigators above. This approval is granted for 12 months and will be reviewed at its anniversary date.

(Signature of Department Head/Administrative Officer/Research Director for the

(Date)

Emergency Medicine Research Advisory Committee)

(Signature of Department Nursing Administrative Officer/Manager

(Date)

Note:

Attachments

Ethics Review (if approved)

Collaborator agreement

Others:

Study protocol

When completed, return to 


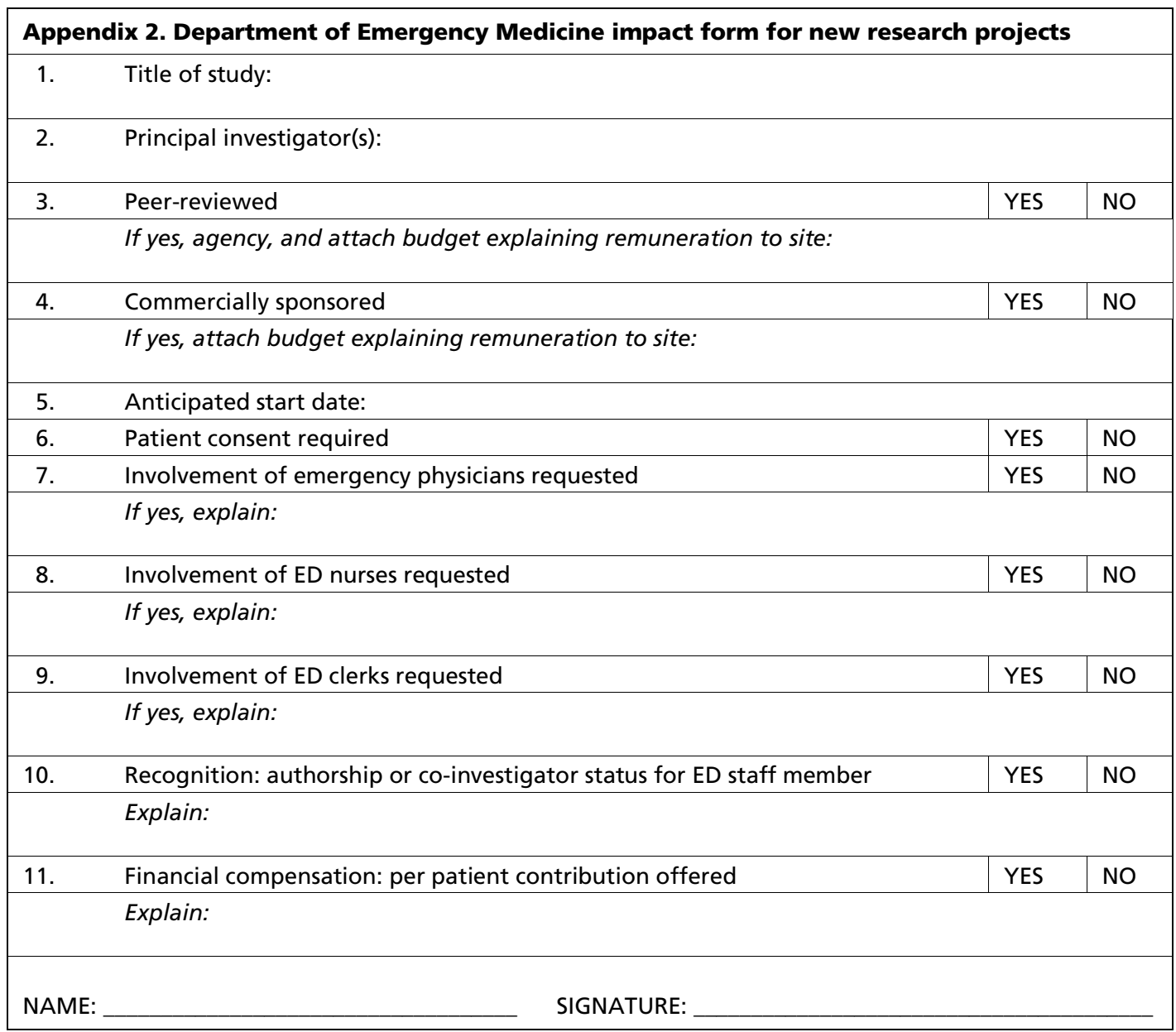

See also Appendix 3, page 248. 


\section{Appendix 3. Policy for Research Studies in the Department of Emergency Medicine}

\section{Introduction}

The Department of Emergency Medicine has a tradition of producing its own high quality original research and of cooperating with the research projects of other hospital departments. This academic mission must be balanced with the demands such research projects place on the other priorities of the department, namely patient care and teaching. Furthermore, multiple simultaneous research projects may conflict with each other and may compete for the same patients as well as for the time and attention of our clerical, nursing, and medical staff. Finally, it is appropriate that the Department of Emergency Medicine receive appropriate recognition and compensation for all research studies that involve Emergency Department patients or staff.

\section{Objective}

To encourage and coordinate research in the Emergency Department of the such that patient care and workload to all staff is not adversely affected.

\section{Process}

The Hospital Department of Emergency Medicine Research Committee will review al proposed research projects prior to their implementation in the department. The committee will review the impact on patient care, the demands on Emergency Department staff, the potential conflict with ongoing studies, and the recognition/compensation to be offered.

\section{Procedures}

1. Submit all correspondence to Dr. , Chair of the Department of Emergency Medicine Research Committee.

2. Forward electronically the complete protocol, a protocol summary, and consent form to

3. Complete and submit an electronic version of the attached " Medicine Impact Form for New Research Projects." Department of Emergency

4. Recognition and compensation will be agreed upon prior to commencement of the study.

a) Peer Reviewed Studies

Ideally involvement would start at the time of protocol development and an Emergency Department staff member would usually be recognized as a co-investigator and coauthor. Compensation will be minimal to nil for such studies.

b) Industry Sponsored Studies

Members of the Department do not expect co-authorship. Compensation to either the Emergency Department Research Fund or the Nursing Emergency Department Education Fund would be expected in proportion to the responsibility and work involved for the physician or nursing group respectively. This should also represent fair compensation related to the overall budget for the study.

5. Discussion regarding new projects will occur prior to REB application. Once the study has been reviewed and approved by the Department Research Committee, the Department Impact Section of the REB application will be signed off. 